\title{
An Apriori Algorithm-Based Association Rule Analysis to Identify Acupoint Combinations for Treating Diabetic Gastroparesis
}

\author{
Ping-Hsun Lu $\mathbb{C D}^{1,2}$ Jui-Lin Keng $\mathbb{C}^{3},{ }^{3}$ Fu-Ming Tsai ${ }^{1},{ }^{4}$ Po-Hsuan Lu ${ }^{1},{ }^{5,6}$ \\ and Chan-Yen Kuo iD ${ }^{4}$ \\ ${ }^{1}$ Department of Chinese Medicine, Taipei Tzu Chi Hospital, Buddhist Tzu Chi Medical Foundation, New Taipei City, Taiwan \\ ${ }^{2}$ School of Post-Baccalaureate Chinese Medicine, Tzu Chi University, Hualien, Taiwan \\ ${ }^{3}$ Department of Applied Mathematics, University of Washington, Seattle, WA, USA \\ ${ }^{4}$ Department of Research, Taipei Tzu Chi Hospital, Buddhist Tzu Chi Medical Foundation, New Taipei City, Taiwan \\ ${ }^{5}$ Department of Medicine, Mackay Medical College, New Taipei City, Taiwan \\ ${ }^{6}$ Department of Dermatology, Mackay Memorial Hospital, Taipei, Taiwan
}

Correspondence should be addressed to Po-Hsuan Lu; pohsuan@gmail.com and Chan-Yen Kuo; cykuo863135@gmail.com

Received 4 December 2020; Revised 25 February 2021; Accepted 28 February 2021; Published 26 March 2021

Academic Editor: Youhua Xu

Copyright (C) 2021 Ping-Hsun Lu et al. This is an open access article distributed under the Creative Commons Attribution License, which permits unrestricted use, distribution, and reproduction in any medium, provided the original work is properly cited.

\begin{abstract}
We explored the potential association rules within acupoints in treating diabetic gastroparesis (DGP) using Apriori algorithm complemented with another partition-based algorithm, a frequent pattern growth algorithm. Apriori algorithm is a data miningbased analysis that is widely applied in various fields, such as business and medicine, to mine frequent patterns in datasets. To search for effective acupoint combinations in the treatment of DGP, we implemented Apriori algorithm to investigate the association rules of acupoints among 17 randomized controlled trials (RCTs). The acupoints were extracted from the 17 included RCTs. In total, 29 distinct acupoints were observed in the RCTs. The top 10 frequently selected acupoints were CV12, ST36, PC6, ST25, BL21, BL20, BL23, SP6, BL18, and ST21. The frequency pattern of acupoints achieved by using a frequent pattern growth algorithm also confirms the result. The results showed that the most associated rules were $\{$ BL23, BL18 $\} \geq\{$ SP6 $\},\{B L 20, B L 18\} \geq$ $\{$ PC6 $\},\{$ PC6, BL18 $\} \geq\{$ BL20 $\}$, and $\{$ SP6, BL18 $\} \geq\{$ BL23 $\}$ in the database. Acupoints, including BL23, BL18, SP6, BL20, and PC6, can be deemed as core elements of acupoint combinations for treating DGP.
\end{abstract}

\section{Introduction}

With the changes in lifestyle and the prevalence of obesity, International Diabetes Federation has reported a total of 451 million people with diabetes in the world, and the number of diabetes mellitus (DM) patients may rise to 693 million in 2045. DM is a non-negligible problem in developing countries [1]. Diabetic gastroparesis (DGP) is a common complication of autonomic neuropathy that occurs in patients with hyperglycemia or DM. Furthermore, DGP affects around $4.8 \%$ of patients with type I DM and $1 \%$ of those with type II DM [2]. Nausea, vomiting, early satiety, postprandial fullness, abdominal distension, and abdominal pain are often observed in patients with DGP. Wide glycemic fluctuations and impact on quality of life are also noticed in DGP patients $[3,4]$. The possible pathogenesis of DGP includes multiple factors, such as vagus neuropathy, abnormal myenteric neurotransmission, damage of inhibitory neuronal function, and the impaired function of smooth muscle cells and interstitial Cajal cells (ICCs) [5-7]. The suggested treatment for DGP depends on the severity. For mild-to-moderate patients, recommendations consist of controlling blood sugar, adjusting diet, and using prokinetic agents. For severe patients, gastric electrical stimulation or placement of a feeding jejunostomy to provide nutrition is suggested. Gastrectomy is the last resort for DGP refractory to treatment [5]. Metoclopramide, erythromycin, domperidone, and cisapride are prokinetic agents that are commonly used to treat DGP, but they present short-term efficacy and may have adverse 
effects, including insomnia, extrapyramidal symptoms, and cardiac arrhythmias $[5,8]$. To date, sufficient evidence that supports surgical treatment as beneficial for DGP is still lacking $[8,9]$. Therefore, other alternative treatment options for DGP must be developed.

Acupuncture, an ancient therapy that has been used in China for over 2500 years, has become popular for its adjunctive role to ameliorate certain disease symptoms and to treat gastrointestinal tract disorders with limited side effects [10]. Acupuncture can improve gastric motility in animal models and humans $[11,12]$. The possible mechanisms to improve the dyspeptic symptoms in DGP by acupuncture include restoration of the enteric nervous system (ENS), restoration of the ultrastructure and pacing function of ICCs, calibration of gastrointestinal hormone disorders, and improvement of gastrointestinal motility $[13,14]$. The choice and combination of acupoints have been broadly accepted as important for successful acupuncture treatment. Yellow Emperor's Internal Classic and the ancient Meridian theory serve as guidelines for the selection of acupoints and their combination $[15,16]$. Nevertheless, no consensus has been reached regarding the standard acupoint combinations for DGP treatment.

Data mining methods have been broadly applied in acupuncture. Data mining methods have been applied to evaluate the option and combination of acupuncture to treat reflux esophagitis [17], chronic atrophic gastritis [18], diarrhea [19], and diabetic peripheral neuropathy [20]. Given that acupuncture therapy focuses on numerous acupoints simultaneously in clinical practice, Apriori algorithm-based association rule analysis can be an essential and useful approach to explore the basic rules. Nevertheless, the acupoint combination for DGP treatment based on randomized controlled trials (RCTs) with a high level of evidence remains lacking. Data mining algorithms are frequently used in Chinese medicine and acupuncture combination analyses in the attempt to treat different types of syndromes [21]. Apriori, one of the association rule mining algorithms, proceeds by identifying the frequency of item sets in large databases and then determining their corresponding association rules [22]. Thus, Apriori algorithm provides comprehensive association rule analysis and offers insights into databases. Certain metrics, support, confidence, and lift are specified in Apriori to help us understand the strength of association rules [22]. Frequent pattern growth algorithm, on the other hand, mines the most frequent pattern and presents a dataset in the form of a tree with its structure constructed by the associations between items in the dataset. Similar to Apriori algorithm, it uses basic metrics, support, confidence, lift, and expected confidence to evaluate the frequent patterns [23].

In this paper, we primarily use Apriori algorithm to achieve the results, which are then further compared with the results obtained from the frequent pattern algorithm. We focused on the exploration of the promising core combination of acupoint combination to treat DGP by applying the Apriori association rule learning analysis based on RCTs [24].

\section{Materials and Methods}

2.1. Literature Search. Articles published up to January 2020 were searched for in PubMed, EMBASE, CENTRAL, and clinicaltrials.gov. The following MeSH and Emtree search headings were used: (diabet* AND gastroparesis) AND (acupuncture OR electroacupuncture OR acup*). For different databases, we have adjusted the search terms accordingly. Unpublished studies were sought out from the ClinicalTrials.gov registry (http://clinicaltrials.gov/). Only human studies were included. We extracted data on the acupoints used for DGP treatment from these 17 RCTs, fourteen of which were included in a meta-analysis article (Table 1) [24-27].

2.2. Study Selection. We selected RCTs to assess the efficacy of acupuncture in the treatment of DGP patients. The following inclusion criteria were applied: randomized controlled trials with DGP diagnosis without gastric outlet ulceration or obstruction by radiographic examination, treatment including acupuncture, electroacupuncture (EA), abdomen acupuncture, eye acupuncture, ear acupuncture, scalp acupuncture, acupuncture combined with acupuncture-related auxiliary techniques and with the sham acupuncture, or gastroprokinetic agents as the control groups, and quantified data available for DGP symptoms. The exclusion criteria were the use of moxibustion, transcutaneous electroacupuncture, acupoint injection, acupuncture therapy combined with Chinese materia medica, incomplete data, and quantified data available for dyspeptic symptoms.

2.3. Risk of Bias (RoB) Assessment. Two authors independently assessed the methodological quality of the included studies using the Cochrane Collaboration's RoB tool [18]. Any disagreement between the investigators was resolved by the third author. Several domains were assessed, namely, allocation generation; allocation concealment; blinding of participants, personnel, and outcome assessors; completeness of outcome data; freedom from selective reporting; and freedom from other forms of bias.

2.4. Data Analysis. In this study, software RStudio (version 1.2.5033, Integrated Development for R, RStudio, PBC, Boston, MA) was used to conduct Apriori association rule learning analysis and plot charts [28]. The dataset contains 14 columns, where each column represents individual formulas. The dataset was then fitted by using the $\mathrm{R}$ package "arules." Data visualization of the charts was performed by fitting the dataset into the R package, "arulesViz." In contrast, we utilized Python programming language's opensource package (Python Software Foundation, Python Language Reference, version 3.7), "pyfpgrowth," to conduct a frequent pattern growth algorithm [29].

Association rule learning algorithm is one of the widely used techniques to detect and analyze relations and useful information from transaction data. Numerous studies implemented association rule learning algorithms to identify 
TABLE 1: Summary of 17 RCTs of acupuncture treatment for DGP.

\begin{tabular}{lccc}
\hline Study (year) & Study design & Inclusion criteria & Acupoints \\
\hline Wang et al. (2008) & RCT & DGP & ST36, LI4 \\
Ge et al. (2010) & RCT & DGP & CV12, ST36, PC6 \\
Shen et al. (2010) & RCT & DGP & PC6, CV12, CV6, ST36, SP6 \\
Wang et al. (2010) & RCT & DGP & BL20, BL21, BL18, BL23, PC6, ST36, SP6, CV12 \\
Zeng and Chai (2008) & RCT & DGP & BL21, CV12, BL20, LR13, BL23, BL18, LR14, GB25, ST25, ST36 \\
Zhang et al. (2007) & RCT & DGP & CV12, ST36, PC6 \\
Zheng and Ge (2010) & RCT & DGP & CV12, ST36, PC6 \\
Han et al. (2001) & RCT & DGP & ST36, ST25, PC6, ST39, CV12 \\
Li (2006) & RCT & DGP & ST36, CV12, ST25, BL21, BL20, LR3, BL23, PC6 \\
Wang (2007) & RCT & DGP & BL21, CV12, BL20, LR13, BL23, BL18, LR14, GB25, ST25, ST36 \\
Wang et al. (2009) & RCT & DGP & CV17, CV13, CV12, CV4, CV10, CV8, CV6 \\
Chen (2008) & RCT & DGP & CV12, ST36, ST25, ST21, ST37 \\
Zhao (2011) & RCT & DGP & ST36, CV12, ST25, ST21, ST37 \\
Chen (2005) & RCT & DGP & CV12, ST21, ST25, BL21, ST36 \\
Zhang et al. (2013) & RCT & DGP & CV12, ST36, PC6 \\
Li et al. (2015) & RCT & DGP & CV12, ST36, PC6 \\
Song et al. (2020) & RCT & DGP & CV12, ST36, PC6, SP9, SP10, SP6, SP8, LI11, LI4, ST40, LR3, SP4 \\
\hline RCT High \\
\hline
\end{tabular}

RCT: randomized controlled trial; DGP: diabetic gastroparesis.

medicine compatibility and to find interdependence within medical record data [22]. The association rule learning algorithm contains an antecedent and consequent set, both of which are a set of items.

Support and confidence factors are essential parameters in association rule learning. Support estimates the frequency of an acupoint appearing in the 14 formulas. On the other hand, confidence measures the frequency of acupoint $\mathrm{A}$ appearing in the formula, given that acupoint $\mathrm{B}$ appears simultaneously. Expected confidence is the number of formulas that include the consequent set of acupoints divided by the total number of formulas. Lift is the likelihood of an increase in the consequent given a particular antecedent. Namely, lift is the probability of the acupoint B appearing when acupoint $\mathrm{A}$ is present in a DGP formula given an expected confidence. It equals the confidence divided by expected confidence. During the exploration of the association rules, users need to test multiple combinations of minimum values for support and confidence factors to discover the significant association rules. However, the selection of thresholds showed slight ambiguity and varied from case to case. If the parameter thresholds were set at extremely high values, then certain meaningful information would be discarded.

In this study, the minimum thresholds for support and confidence factors were $20 \%$ and $60 \%$, respectively. The highest value of the support factor was 0.6 , and by increasing the minimum value of support from 0.1 to 0.2 , we can filter out 82 rules down to 19 rules given that the confidence's minimum value was set to 0.6 . The association rules were sorted by support factor in descending order.

\section{Results}

3.1. Study Characteristics. The acupuncture treatment for DGP used an average of five acupoints, and the average duration of treatment was 22 days. In the meta-analysis, compared with the control group, acupuncture therapy showed a higher response rate (RR, 1.20 (95\% confidence interval (CI), 1.12 to 1.29$), P<0.00001$ ) and significantly improved dyspeptic symptoms like stomach fullness, loss of appetite, and nausea and vomiting in the treatment group, but there is no real difference between the two groups of gastric emptying [24]. Li et al. reported that in patients with diabetic gastroparesis, acupuncture reduces gastric retention, improves gastroparesis symptoms [25], and is effective for epigastric fullness [27]. Song et al. showed acupuncture treatment improved the DGP symptom score and reduced serum content of transmembrane protein 16A (a selective marker of ICC) [26].

3.2. RoB Assessment. Table 1 summarizes the methodological quality of the included studies, whereas Supplementary Figure 1 provides the detailed RoB assessment. The 17 RCTs exhibited variable overall quality. This finding can be possibly explained by one of the following reasons. The random sequences of 11 studies out of 17 trials were correct and had apparent descriptions [24-27]. None of the retrieved studies gave a detailed description of allocation concealment, only two trials used patient blinding with sham electroacupuncture [24, 25], seven studies reported incomplete data, and seven studies did not give the baseline comparison of the severity of DGP symptoms [24].

3.3. Acupoint Distribution. We identified 29 acupoints from the 17 RCTs on acupuncture treatment for DGP. Figure 1 shows the frequency distribution of acupoints. The 10 most frequently selected acupoints for treating DGP were CV12, ST36, PC6, ST25, BL21, BL20, BL23, SP6, BL18, and ST21.

3.4. Apriori Algorithm-Based Association Rule Analysis for Item Sets of Herb Combinations. We investigated 11 association rules in accordance with the 17 acupoint formulas for 


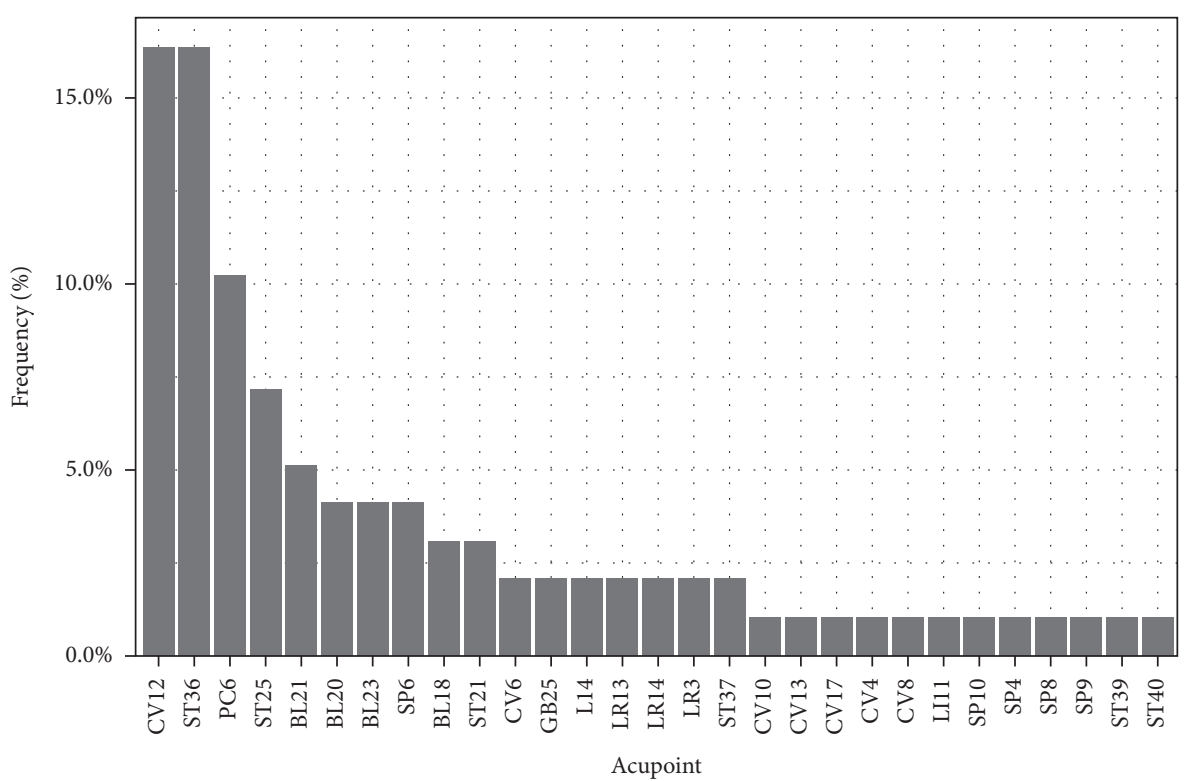

FIGURE 1: Frequency distribution of acupoints used in the 17 RCTs of acupuncture treatment for DGP.

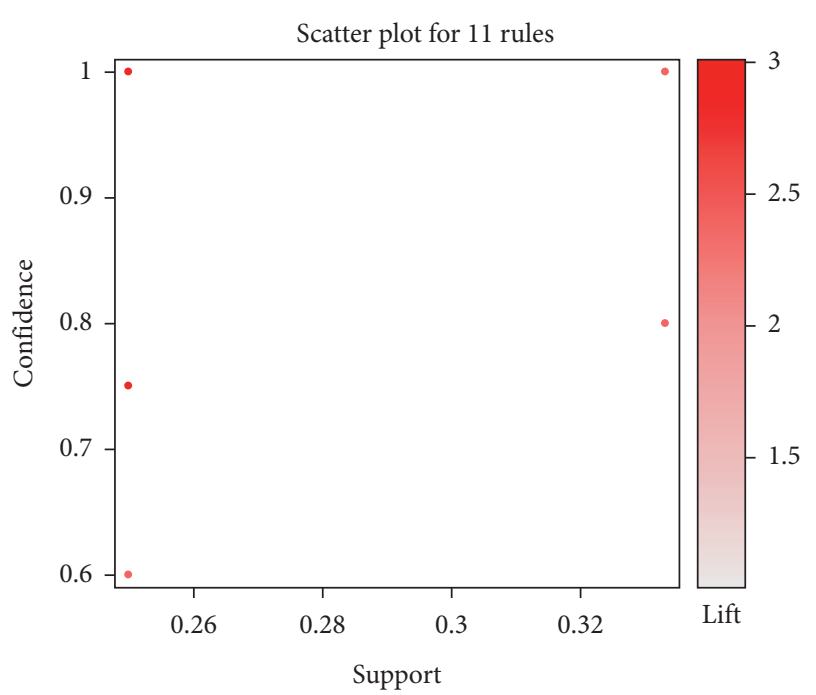

FIgURE 2: Scatter plot for the 11 association rules obtained in the 17 RCTs of acupuncture treatment for DGP via an Apriori algorithmbased association rule analysis.

treating DGP. The association rules were shown based on the scatter plot with support on the $x$-axis and confidence on the $y$-axis. The color of each association rule was decided by the metric's corresponding value and lift (Figure 2). The results demonstrated that the lift values of 11 association rules were larger than 1. Thus, the likelihood of the antecedent and consequent acupoints being selected in the same formula was statistically significantly larger than that of consequent acupoint being selected alone. The metric values for the confidence of all 11 association rules were at least larger than 0.6. Therefore, for each rule, the likelihood of the consequent acupoint being selected when antecedent acupoints are selected is relatively high. By contrast, the metric values for support ranged from 0.2 to 0.6 , indicating the frequency of each individual antecedent acupoint appearing in the formula. This finding suggests the presence of a core list of acupoints to select from for the treatment of DGP. All 11 association rules were ordered by the metric and support (Table 2).

A grouped matrix diagram was used to cluster similar rules into groups (circles) and display the general distribution of association rules (Figure 3). Six clusters were on the horizontal ordinate, and the vertical ordinate represented the acupoints generated by these six clusters (rules). The darker the color of a circle was, the higher was the degree of lift of a group. The size of a circle represents the degree of support. Thus, the larger the circle was, the higher was the degree of support. Figure 4 shows the network graph visualization. The figure shows the 11 association rules found among the 17 acupoint formulas. Acupoints ST36 and CV12 were most likely to be used in association with other acupoints.

3.5. Apriori Algorithm-Based Association Rule Analysis for Item Sets of Herb Combinations Complemented with Frequent Pattern Growth Algorithm. Table 3 shows the frequent patterns of acupoint used in the 17 RCTs based on frequent pattern growth algorithm, and its results are coherent with the result in Figure 4. However, the values of lift to the association rules containing ST36 and CV12 were low, and thus they were not selected in the following result. Acupoints $\{$ BL23, BL18 $\} \geq\{$ SP6 $\},\{$ BL20, BL18 $\} \geq\{$ PC6 $\},\{$ PC6, BL18 $\} \geq$ $\{$ BL20 $\}$, and $\{$ SP6, BL18 $\} \geq\{$ BL23 $\}$ were selected, revealing item sets of antecedent and consequent acupoints. The results of selected association rules matched the association rule $\# 8 \quad\{$ BL23 $\} \geq\{$ SP6 $\}, \# 3 \quad\{$ BL20 $\} \geq\{$ PC6 $\}, \# 4 \quad\{$ PC6 $\} \geq$ $\{$ BL20 $\}$, and $\# 9\{$ SP6 $\} \geq\{$ BL23 $\}$ in Table 2. Likewise, the results of selected association rules are consistent with \#17 ("PC6," "SP6") $\geq$ (“CV12," "ST36"), \#19 (CV12, SP6) $\geq$ (PC6, ST36), \# 6 (BL18, CV12) $\geq$ (BL20, BL21, BL23), \#9 (BL23, CV12) $\geq($ BL20, BL21), \#4 (BL20, BL21) $\geq($ BL23, 
TABLE 2: Apriori algorithm-based association rules for acupoints used in the 17 RCTs on acupuncture treatment for DGP.

\begin{tabular}{|c|c|c|c|c|c|}
\hline No. & Association rules & Support & Confidence & Expected confidence & Lift \\
\hline 1 & $\{$ PC6 $\} \geq\{$ CV 12$\}$ & 0.3333333 & 1.0000000 & 0.4166667 & 2.400000 \\
\hline 2 & $\{\mathrm{CV} 12\} \geq\{$ PC6 $\}$ & 0.3333333 & 0.8000000 & 0.4166667 & 2.400000 \\
\hline 3 & $\{$ BL20 $\} \geq\{$ PC6 $\}$ & 0.2500000 & 1.0000000 & 0.3333333 & 3.000000 \\
\hline 4 & $\{$ PC6 $\} \geq\{$ BL20 $\}$ & 0.2500000 & 0.7500000 & 0.3333333 & 3.000000 \\
\hline 5 & $\{$ BL20 $\} \geq\{\mathrm{CV} 12\}$ & 0.2500000 & 1.0000000 & 0.4166667 & 2.400000 \\
\hline 6 & $\{\mathrm{CV} 12\} \geq\{\mathrm{BL} 20\}$ & 0.2500000 & 0.6000000 & 0.4166667 & 2.400000 \\
\hline 7 & $\{$ BL 21$\} \geq\{$ ST36 $\}$ & 0.2500000 & 1.0000000 & 0.5000000 & 2.000000 \\
\hline 8 & $\{$ BL23 $\} \geq\{$ SP6 $\}$ & 0.2500000 & 1.0000000 & 0.3333333 & 3.000000 \\
\hline 9 & $\{\mathrm{SP} 6\} \geq\{\mathrm{BL} 23\}$ & 0.2500000 & 0.7500000 & 0.3333333 & 3.000000 \\
\hline 10 & $\{$ SP6 $\} \geq\{$ ST36 $\}$ & 0.2500000 & 0.7500000 & 0.6666667 & 1.500000 \\
\hline 11 & $\{\mathrm{CV} 12\} \geq\{\mathrm{ST} 36\}$ & 0.2500000 & 0.6000000 & 0.8333333 & 1.200000 \\
\hline
\end{tabular}

DGP: diabetic gastroparesis.

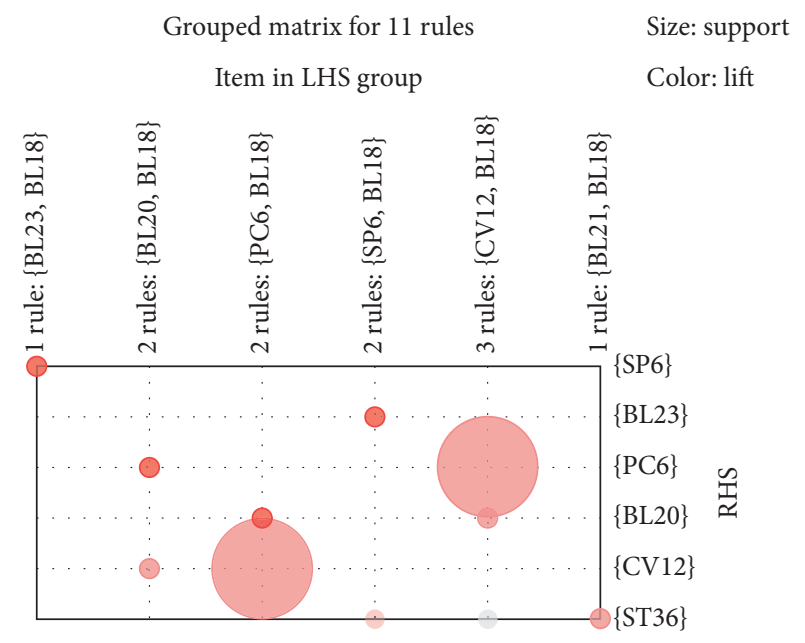

FIGURE 3: Grouping matrix for the 11 association rules obtained in the 17 RCTs of acupuncture treatment for DGP via an Apriori algorithm-based association rule analysis.

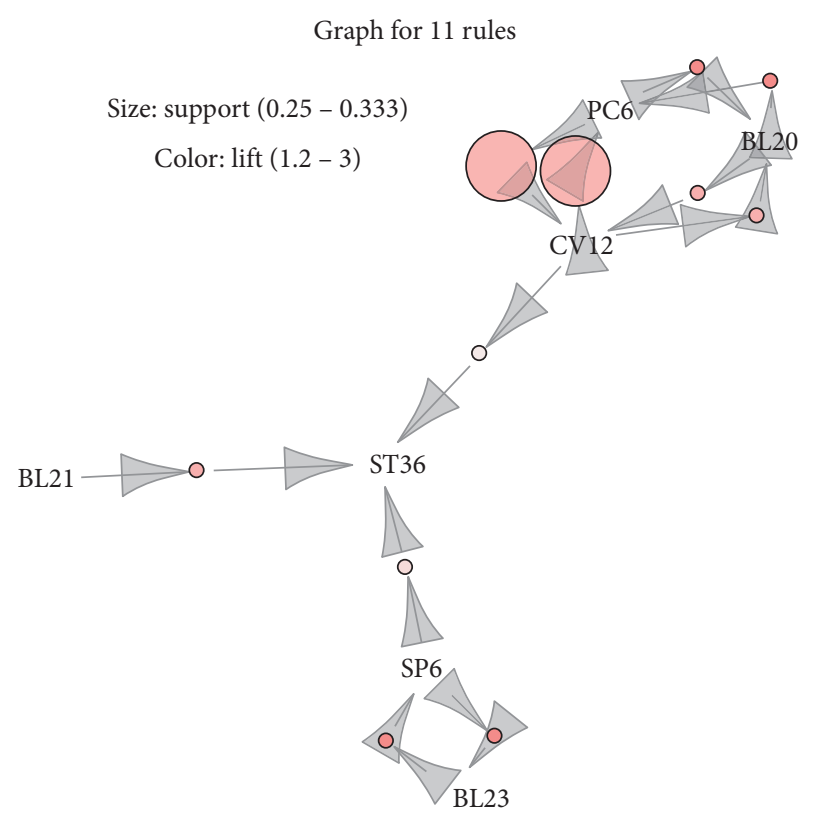

Figure 4: Combination matrix of association rules obtained in the 17 RCTs of acupuncture treatment for DGP via an Apriori algorithm-based association rule analysis.
TABle 3: Frequent patterns of acupoints used in the 17 RCTs on acupuncture treatment for DGP.

\begin{tabular}{|c|c|c|}
\hline No. & Frequent pattern & Support \\
\hline 1 & (“CV12,” “ST36”) & 1.071429 \\
\hline 2 & (“CV12," "PC6") & 0.714286 \\
\hline 3 & (“PC6,” “ST36”) & 0.714286 \\
\hline 4 & (“CV12," “ST25”) & 0.500000 \\
\hline 5 & (“ST25,” “ST36”) & 0.500000 \\
\hline 6 & (“BL21," "ST36”) & 0.357143 \\
\hline 7 & (“BL21," “CV12”) & 0.357143 \\
\hline 8 & (“BL23," “ST36”) & 0.285714 \\
\hline 9 & (“BL23," “CV12”) & 0.285714 \\
\hline 10 & (“BL21," “BL23”) & 0.285714 \\
\hline 11 & (“BL20,” 'BL23”) & 0.285714 \\
\hline 12 & (“BL20," “ST36”) & 0.285714 \\
\hline 13 & (“BL20," “CV12”) & 0.285714 \\
\hline 14 & (“BL20," “BL21”) & 0.285714 \\
\hline 15 & (“CV12," “SP6”) & 0.285714 \\
\hline 16 & (“SP6,” “ST36”) & 0.285714 \\
\hline 17 & (“PC6,” “SP6”) & 0.285714 \\
\hline 18 & (“CV12," “ST21”) & 0.214286 \\
\hline 19 & (“ST21," "ST36”) & 0.214286 \\
\hline 20 & (“ST21,” “ST25”) & 0.214286 \\
\hline 21 & (“BL18," “ST36”) & 0.214286 \\
\hline 22 & (“BL18," “CV12”) & 0.214286 \\
\hline 23 & (“BL18," “BL21”) & 0.214286 \\
\hline 24 & ("BL18," "BL20") & 0.214286 \\
\hline 25 & (“BL18," "BL23") & 0.214286 \\
\hline
\end{tabular}

DGP: diabetic gastroparesis.

ST36), \#5 (BL21, BL23) $\geq($ BL20, ST36), \#17 (PC6, $\mathrm{SP} 6) \geq(\mathrm{CV} 12, \mathrm{ST} 36)$, and $\# 7(\mathrm{BL} 21, \mathrm{CV} 12) \geq(\mathrm{ST} 36)$ in Table 4.

\section{Discussion}

This study indicated BL23, BL18, and SP6 and BL20, BL18, and PC6 as the major acupoint combinations in treating DGP, as confirmed by Apriori association-mining analysis (Figure 5). RCTs of acupuncture for DGP revealed that the acupoint combination played a role in relieving dyspeptic symptoms in patients with DGP.

The evidence-based strategies help to ascertain the efficacy of selecting acupoints for further treatment. To the 
TABLE 4: 24 frequent pattern growth algorithm-based association rules obtained in the 17 RCTs on acupuncture treatment for DGP.

\begin{tabular}{|c|c|c|c|}
\hline No. & LHS & RHS & Confidence \\
\hline 1 & (BL18, BL20) & (BL21, BL23, ST36) & 1.00 \\
\hline 2 & (BL18, BL23) & (BL20, BL21, ST36) & 1.00 \\
\hline 3 & (BL20, BL23) & (BL21, ST36) & 1.00 \\
\hline 4 & (BL18, BL21) & (BL20, BL23, ST36) & 1.00 \\
\hline 5 & (BL20, BL21) & (BL23, ST36) & 1.00 \\
\hline 6 & (BL21, BL23) & (BL20, ST36) & 1.00 \\
\hline 7 & (BL18, CV12) & (BL20, BL21, BL23) & 1.00 \\
\hline 8 & (BL21, CV12) & (ST36) & 0.60 \\
\hline 9 & (BL20, CV12) & (BL21, BL23) & 1.00 \\
\hline 10 & (BL23, CV12) & (BL20, BL21) & 1.00 \\
\hline 11 & (BL18, ST36) & (BL20, BL21, BL23) & 1.00 \\
\hline 12 & (BL21, ST36) & $(\mathrm{CV} 12)$ & 0.60 \\
\hline 13 & (BL20, ST36) & (BL21, BL23) & 1.00 \\
\hline 14 & (BL23, ST36) & (BL20, BL21) & 1.00 \\
\hline 15 & (ST21, ST25) & (CV12) & 1.00 \\
\hline 16 & (ST21, ST36) & (ST25) & 1.00 \\
\hline 17 & (CV12, ST21) & (ST25) & 1.00 \\
\hline 18 & (PC6, SP6) & (CV12, ST36) & 0.75 \\
\hline 19 & (SP6, ST36) & (CV12, PC6) & 0.75 \\
\hline 20 & (CV12, SP6) & (PC6, ST36) & 0.75 \\
\hline 21 & (CV12, ST25) & (ST36) & 1.00 \\
\hline 22 & (ST25, ST36) & (CV12) & 1.00 \\
\hline 23 & (CV12, PC6) & (ST36) & 1.00 \\
\hline 24 & (CV12, ST36) & (PC6) & 0.66 \\
\hline
\end{tabular}

DGP: diabetic gastroparesis.

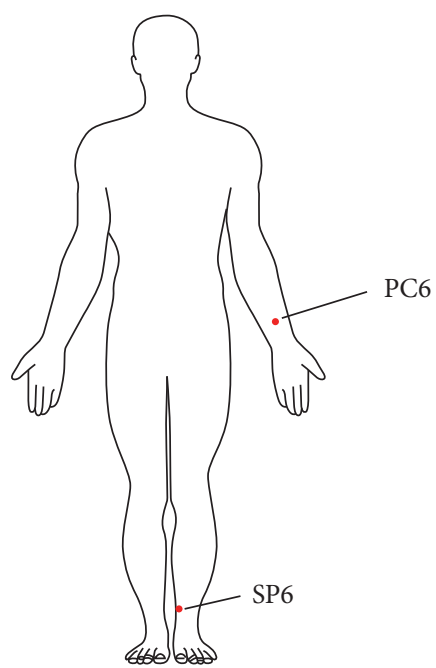

Front

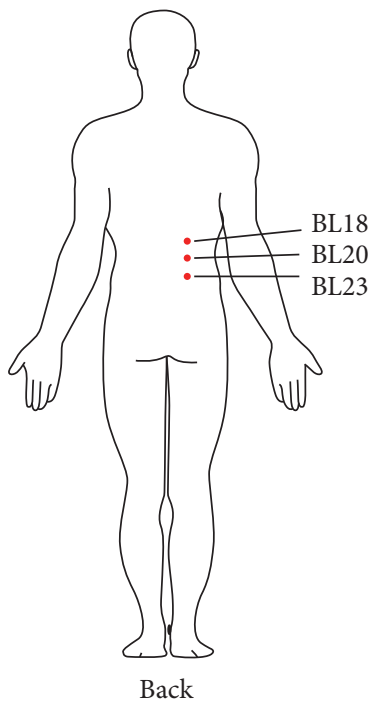

(b)

Figure 5: Location of core acupoints derived from association rules obtained in the 17 RCTs of acupuncture treatment for DGP.

best of our knowledge, this study is the first report on potential core acupoint combinations to treat patients with DGP based on RCTs and data mining analysis. The core acupoint combinations are helpful for patients with DGP; the possible fundamental mechanisms include restoration of ICC $[13,30]$, mediation of hormones, restoration of the ENS, and enhancement of gastrointestinal motility [14, 31]. Stimulating ST36 and CV12 points of the DGP rat with EA can reduce ICC apoptosis and restore ICC structure and pacing function, possibly through the mouse stem cell factor (SCF)/KIT-ETV1 signaling pathway to upregulate the mRNA of SCF and neuronal nitric oxide synthase [32-34]. Hou et al. reported an RCT that included 40 postoperative patients with gastrointestinal tumors; this RCT showed that electrical stimulation on acupoints ST36 and ST37 can elevate the serum levels of gastrin and motilin and 
TABLe 5: Potential efficacy of the core acupoints for DGP treatment.

\begin{tabular}{|c|c|c|c|c|}
\hline Point & $\begin{array}{l}\text { Chinese } \\
\text { name }\end{array}$ & English name & $\begin{array}{l}\text { Primary } \\
\text { meridians }\end{array}$ & Efficacy \\
\hline BL18 [49] & Ganshu & Liver locus & Bladder & $\begin{array}{l}\text { Activation of CRH-like neurons; modulation of the expressions of CRH and } \\
\text { GR in the PVN }\end{array}$ \\
\hline BL20 [54] & Pishu & Spleen locus & Bladder & Suppresses acid secretion and increases $\beta$-EP and SS \\
\hline BL23 [49] & Shenshu & Kidney locus & Bladder & $\begin{array}{l}\text { Activation of CRH-like neurons; modulation of the expressions of CRH and } \\
\text { GR in the PVN }\end{array}$ \\
\hline $\begin{array}{l}\text { SP6 } \\
{[50-52]}\end{array}$ & Sanyinjiao & $\begin{array}{l}\text { Crossroad at } \\
\text { three Yin }\end{array}$ & Spleen & $\begin{array}{l}\text { Protection of ICCs, amelioration of vascular endothelial dysfunction, and } \\
\text { modulation of ghrelin }\end{array}$ \\
\hline $\begin{array}{l}\text { PC6 } \\
{[54-56]}\end{array}$ & Neiguan & Inner pass & Pericardium & $\begin{array}{c}\text { Suppresses acid secretion, increases } \beta \text {-EP and SS, modulates vagovagal } \\
\text { neurocircuits, and decreases the number of double-labeled OT neurons and } \\
\qquad \text {-fos neurons }\end{array}$ \\
\hline
\end{tabular}

DGP: diabetic gastroparesis; CRH: corticotropin-releasing hormone; GR: glucocorticoid receptor; PVN: paraventricular nucleus of the hypothalamus; $\beta$-EP; beta-endorphin; SS: somatostatin; GD: gastric distention; ICCs: interstitial Cajal cells; OT: oxytocin.

electrogastrogram frequency. Additionally, the recovery of gastrointestinal function and reduction of complications, such as flatulence, abdominal pain, and diarrhea, were noted [35]. Jang et al. reported that stimulation with acupoint ST36 in mice can promote the motility of the small intestine via the decrease in serum levels of vasoactive intestinal peptide and an increase in serum levels of motilin, ghrelin, and gastrin [36]. Liang et al. demonstrated that electrical stimulation on acupoints ST36 and ST25 of a constipation mouse model can improve gastrointestinal motility by regulating excitatory and inhibitory neurons in ENS [37]. High-frequency electrical stimulation of ST36 in diabetic rats can promote the regeneration of lost enteric neurons through glial cell-derived neurotrophic factor and phosphatidylinositol-3 kinase/Akt signaling pathways [38].

The combination of acupoints was reported to exhibit greater therapeutic efficacy than a single acupoint $[39,40]$. Wang et al. reported that the combination of multiple acupoints resulted in improved the score of the Pittsburgh sleep quality index and Athens Insomnia Scale via regulation of the activity of the brain area related to sleep experience [39]. The RCT which enrolled 30 patients with hypertension showed that the combined acupuncture points LR3 and KI3 can activate a wider area of the brain than a single point acupuncture based on the resting-state functional magnetic resonance imaging scan [41]. Gao et al. demonstrated that stimulating acupoint combination in slow transit constipation mice showed higher defecation grain number and intestinal propulsion rate compared with a single acupoint [42]. In DGP model rats, stimulation of the combined acupoints ST36 and CV12 can produce more ICCs than the single point ST36 [43]. Hence, an acupoint combination displays better pharmacological efficacy than a single acupoint. Even though there are also articles discussing the compatibility laws of acupuncture in the treatment of DGP, it uses fewer databases, not selects a higher level of evidence article, such as RCT, and does not elaborate on the association analysis $[44,45]$. We implemented systemic review and Apriori algorithm to investigate the association rules of acupoints among 17 RCTs to demonstrate the effectiveness of acupuncture on patients with DGP. Moreover, research studies indicate that the core acupoint combinations we discovered can improve DGP through the protection of ICCs, amelioration of vascular endothelial dysfunction, hormone modulation, and stimulation of neurons [46-53] (Table 5).

4.1. Limitation. Despite our results on potential core acupoint combinations for the treatment of DGP, our study has several limitations. First, most RCTs included in this meta-analysis revealed a high RoB, and only two studies used the blinding method. Thus, a high RoB should be considered to explain these results. Second, most RCTs lacked a careful follow-up. The treatment duration was 22 days on average in this meta-analysis. Therefore, further research should be carried out regarding the long-term efficacy and safety of these core acupoint combinations for DGP. Third, as the mechanisms of action of acupoint combinations are unclear, more basic and clinical studies are required for a comprehensive assessment.

\section{Conclusions}

Based on RCTs and data mining analysis, the acupoint combinations of BL23, BL18, and SP6 and BL20, BL18, and PC6 are potential acupuncture treatments for DGP. The acupoint combinations also significantly improved stomach fullness, nausea and vomiting, and loss of appetite. However, further trials with larger sample sizes and appropriate controls are recommended.

\section{Data Availability}

The data utilized to support the findings of this study are included within the article.

\section{Conflicts of Interest}

The authors declare that there are no conflicts of interest regarding the publication of this article. 


\section{Acknowledgments}

The authors thank Taipei Tzu Chi Hospital and the participants for their contribution to the study. This study was supported by Taipei Tzu Chi Hospital, Buddhist Tzu Chi Medical Foundation, New Taipei City, Taiwan (TCRD-TPE109-12, TCRD-TPE-109-06(1/2), and TCRD-TPE-110-49).

\section{Supplementary Materials}

Supplementary Figure 1: RoB assessment of the retrieved RCTs. (Supplementary Materials)

\section{References}

[1] N. H. Cho, J. E. Shaw, S. Karuranga et al., "IDF diabetes atlas: global estimates of diabetes prevalence for 2017 and projections for 2045," Diabetes Research and Clinical Practice, vol. 138, pp. 271-281, 2018.

[2] B. Moshiree, M. Potter, and N. J. Talley, "Epidemiology and pathophysiology of gastroparesis," Gastrointestinal Endoscopy Clinics of North America, vol. 29, no. 1, pp. 1-14, 2019.

[3] S. Krishnasamy and T. L. Abell, "Diabetic gastroparesis: principles and current trends in management," Diabetes Therapy, vol. 9, no. 1, pp. 1-42, 2018.

[4] W. A. Hoogerwerf, P. J. Pasricha, A. N. Kalloo, and M. M. Schuster, "Pain: the overlooked symptom in gastroparesis," American Journal of Gastroenterology, vol. 94, no. 4, pp. 1029-1033, 1999.

[5] G. S. Aswath, L. A. Foris, A. K. Ashwath, and K. Patel, Diabetic Gastroparesis, StatPearls Publishing LLC., Treasure Island, FL, USA, 2020.

[6] S. S. Yarandi and S. Srinivasan, "Diabetic gastrointestinal motility disorders and the role of enteric nervous system: current status and future directions," Neurogastroenterology \& Motility, vol. 26, no. 5, pp. 611-624, 2014.

[7] K. Kishi, N. Kaji, T. Kurosawa, S. Aikiyo, and M. Hori, "Hyperglycemia in the early stages of type 1 diabetes accelerates gastric emptying through increased networks of interstitial cells of Cajal," PLoS One, vol. 14, no. 10, p. e0222961, 2019.

[8] H. P. Parkman, W. L. Hasler, and R. S. Fisher, "American gastroenterological association technical review on the diagnosis and treatment of gastroparesis," Gastroenterology, vol. 127, no. 5, pp. 1592-1622, 2004.

[9] M. P. Jones and K. Maganti, "A systematic review of surgical therapy for gastroparesis," American Journal of Gastroenterology, vol. 98, no. 10, pp. 2122-2129, 2003.

[10] “Acupuncture NCDPo. Acupuncture," JAMA, vol. 280, no. 17, pp. 1518-1524, 1998.

[11] J. Chen, G.-Q. Song, J. Yin, T. Koothan, and J. D. Z. Chen, "Electroacupuncture improves impaired gastric motility and slow waves induced by rectal distension in dogs," American Journal of Physiology-Gastrointestinal and Liver Physiology, vol. 295, no. 3, pp. G614-G620, 2008.

[12] J. Yin and J. D. Chen, "Gastrointestinal motility disorders and acupuncture," Autonomic Neuroscience, vol. 157, no. 1-2, pp. 31-37, 2010.

[13] Y. Gao, G. Lu, Y. Y. Wang, L. Chen, R. Chen, and F. X. Liang, "[Prospects and progress on the mechanisms of acupuncture underlying improvement in diabetic gastroparesis]," Zhen $\mathrm{Ci}$ Yan Jiu, vol. 42, no. 4, pp. 367-371, 2017.
[14] Y. Feng, Y. Fang, Y. Wang, and Y. Hao, “Acupoint therapy on diabetes mellitus and its common chronic complications: a review of its mechanisms," BioMed Research International, vol. 2018, Article ID 3128378, 9 pages, 2018.

[15] W. Zhou and P. Benharash, "Effects and mechanisms of acupuncture based on the principle of meridians," Journal of Acupuncture and Meridian Studies, vol. 7, no. 4, pp. 190-193, 2014.

[16] W. B. Zhang, Y. P. Wang, and H. Y. Li, "[Analysis on correlation between meridians and viscera in book the yellow emperor's internal classic]," Zhen Ci Yan Jiu, vol. 43, no. 7, pp. 424-429, 2018.

[17] Y. Yi, L. X. Pei, H. Chen et al., "[Analysis on acupoint selection rules for reflux esophagitis]," Zhongguo Zhen Jiu, vol. 40, no. 5, pp. 557-564, 2020.

[18] Y. Liu, H. Gong, J. Liu, and H. Zhang, "[Acupoint selection pattern of chronic atrophic gastritis based on data mining methods of latent structure model and frequency item set]," Zhongguo Zhen Jiu, vol. 38, no. 6, pp. 667-671, 2018.

[19] Z. W. Su, Y. L. Ren, S. Y. Zhou et al., "[Analysis on characteristics of meridians and acupoints of acupuncture and moxibustion for diarrhea in ancient based on data mining]," Zhongguo Zhen Jiu, vol. 33, no. 10, pp. 905-909, 2013.

[20] H. Pan, H. Wang, Y. Wang, and H. Huang, "[Rules of acupoint selection for diabetic peripheral neuropathy based on data mining technology]," Zhongguo Zhen Jiu, vol. 36, no. 10, pp. 1111-1114, 2016.

[21] P.-H. Lu, J.-L. Keng, K.-L. Kuo, Y.-F. Wang, Y.-C. Tai, and C.-Y. Kuo, "An Apriori algorithm-based association rule analysis to identify herb combinations for treating uremic pruritus using Chinese herbal bath therapy," Evidence-Based Complementary and Alternative Medicine, vol. 2020, Article ID 8854772, 9 pages, 2020.

[22] R. J. Bayardo Jr. and R. Agrawal, "Mining the most interesting rules," in Proceedings of the Fifth ACM SIGKDD International Conference on Knowledge Discovery and Data Mining, San Diego, CA, USA, August 1999.

[23] J. Han and J. Pei, "Mining frequent patterns by patterngrowth: methodology and implications," ACM SIGKDD Explorations Newsletter, vol. 2, no. 2, pp. 14-20, 2000.

[24] M. Yang, X. Li, S. Liu et al., "Meta-analysis of acupuncture for relieving non-organic dyspeptic symptoms suggestive of diabetic gastroparesis," BMC Complementary Medicine and Therapies, vol. 13, p. 311, 2013.

[25] G. Li, C. Huang, X. Zhang et al., "The short-term effects of acupuncture on patients with diabetic gastroparesis: a randomised crossover study," Acupuncture in Medicine, vol. 33, no. 3, pp. 204-209, 2015.

[26] Y. J. Song, X. Wang, X. J. Li, and Z. L. Zhang, "[Effect of Tiaoli Piwei needling technique on diabetic gastroparesis and transmembrane protein 16A]," Zhongguo Zhen Jiu, vol. 40, no. 8, pp. 811-815, 2020.

[27] B. M. Zhang, Z. H. Hu, Y. Shou, Y. Wang, S. W. Xu, and Y. Yang, "Treatment of diabetic gastroparesis by acupuncture therapy: a multi-center randomized controlled trial," Shanghai Journal of Traditional Chinese Medicine, vol. 47, no. 3, pp. 31-34, 2013.

[28] J. Verzani, Getting Started with RStudio:, O'Reilly Media, Inc., Newton, MA, USA, 2011.

[29] R. Gv and F. L. Drake, The Python Language Reference Manual, Network Theory Ltd., London, UK, 2011.

[30] X. Wei, Y. Lin, D. Zhao et al., "Electroacupuncture relieves suppression of autophagy in interstitial cells of cajal of diabetic 
gastroparesis rats," Canadian Journal of Gastroenterology and Hepatology, vol. 2020, Article ID 7920715, 10 pages, 2020.

[31] X. F. Wu, X. L. Chen, X. N. Zheng et al., "[Effect of different stimulating strength of electroacupuncture on gastrointestinal motility and RhoA/ROCK signaling in gastric antral smooth muscle in diabetic gastroparesis rats]," Zhen Ci Yan Jiu, vol. 43, no. 3, pp. 169-174, 2018.

[32] C.-C. Zhang, Y.-P. Lin, Y. Peng et al., "Study on the mechanisms of electroacupuncture for promoting gastrointestinal motility in rats with diabetic gastroparesis," Journal of Acupuncture and Tuina Science, vol. 15, no. 3, pp. 158-164, 2017.

[33] L. Tian, B. Zhu, and S. Liu, "Electroacupuncture at ST36 protects ICC networks via $\mathrm{mSCF} / \mathrm{kit}-\mathrm{ETV} 1$ signaling in the stomach of diabetic mice," Evidence-based Complementary and Alternative Medicine, vol. 2017, Article ID 3980870, 13 pages, 2017.

[34] C. C. Zhang, Y. P. Lin, Y. Peng et al., "[Effects of electroacupuncture on ultrastructure of interstitial cells of cajal and stem cell factor-kit signal pathway of gastric antrum in diabetic gastroparesis rats]," Zhen Ci Yan Jiu, vol. 42, no. 6, pp. 482-488, 2017.

[35] L. Hou, L. Xu, Y. Shi, and F. Gu, "Effect of electric acupoint stimulation on gastrointestinal hormones and motility among geriatric postoperative patients with gastrointestinal tumors," Journal of Traditional Chinese Medicine, vol. 36, no. 4, pp. 450-455, 2016.

[36] J.-H. Jang, D.-J. Lee, C.-H. Bae et al., "Changes in small intestinal motility and related hormones by acupuncture stimulation at Zusanli (ST 36) in mice," Chinese Journal of Integrative Medicine, vol. 23, no. 3, pp. 215-220, 2017.

[37] C. Liang, K. Y. Wang, M. R. Gong, Q. Li, Z. Yu, and B. Xu, "Electro-acupuncture at ST37 and ST25 induce different effects on colonic motility via the enteric nervous system by affecting excitatory and inhibitory neurons," Neurogastroenterologu and Motility, vol. 30, no. 7, p. e13318, 2018.

[38] F. Du and S. Liu, "Electroacupuncture with high frequency at acupoint ST-36 induces regeneration of lost enteric neurons in diabetic rats via GDNF and PI3K/AKT signal pathway," American Journal of Physiology-Regulatory, Integrative and Comparative Physiology, vol. 309, no. 2, pp. R109-R118, 2015.

[39] Y. K. Wang, T. Li, L. J. Ha et al., "Effectiveness and cerebral responses of multi-points acupuncture for primary insomnia: a preliminary randomized clinical trial and fMRI study," $B M C$ Complementary Medicine and Therapies, vol. 20, no. 1, p. 254, 2020.

[40] P. C. Hsieh, C. F. Cheng, C. W. Wu et al., "Combination of acupoints in treating patients with chronic obstructive pulmonary disease: an apriori algorithm-based association rule analysis," Evidence-Based Complementary and Alternative Medicine, vol. 2020, Article ID 8165296, 7 pages, 2020.

[41] J. Zhang, X. Cai, Y. Wang et al., "Different brain activation after acupuncture at combined acupoints and single acupoint in hypertension patients: an rs-fMRI study based on ReHo analysis," Evidence-Based Complementary and Alternative Medicine, vol. 2019, Article ID 5262896, 10 pages, 2019.

[42] F. Gao, H. M. Sheng, T. N. Zhang, S. B. Wu, J. Cao, and M. Q. Zhou, "[Effect of manual acupuncture stimulation of single acupoint or acupoints combination on intestinal movement in slow transit constipation mice]," Zhen Ci Yan Jiu, vol. 42, no. 1, pp. 62-66, 2017.

[43] Y. Q. Li, B. Yu, T. Li, Z. J. Zhao, M. J. Yang, and F. C. Wang, "Effect of acupuncture at single acupoint and combined acupoints on antral interstitial cells of cajal in diabetic gastroparesis model rats," World Chinese Medicine, vol. 11, no. 2, pp. 214-218, 2016.

[44] F. Cao, T. Li, L. J. Ha, C. X. Shan, M. J. Zhi, and F. C. Wang, "[Analysis of compatibility laws for acupoint selection of acupuncture in treating diabetic gastroparasis]," Zhongguo Zhong Xi Yi Jie He Za Zhi, vol. 36, no. 5, pp. 549-552, 2016.

[45] P. Li, Y. Z. Hui, Q. Q. Wen, and Y. Wang, "Analysis of acupoint selection rules to treat diabetic gastroparesis with acupuncture based on the clinical controlled trials," Journal of Clinical Acupuncture and Moxibustion, vol. 31, no. 6, pp. 37-39, 2015.

[46] E. Siegel, The Acupuncture Point Functions Charts and Workbook, Jessica Kingsley Publishers, London, UK, 2019.

[47] C. Liang, Y. Wang, B. Xu, and Z. Yu, "[Effect of acupuncture at different acupoints on electric activities of rat cerebellar fastigial nuclear]," Zhongguo Zhong Xi Yi Jie He Za Zhi, vol. 35, no. 4, pp. 476-480, 2015.

[48] H. Wang, W. J. Liu, G. M. Shen, M. T. Zhang, S. Huang, and $\mathrm{Y}$. He, "Neural mechanism of gastric motility regulation by electroacupuncture at RN12 and BL21: a paraventricular hypothalamic nucleus-dorsal vagal complex-vagus nervegastric channel pathway," World Journal of Gastroenterology, vol. 21, no. 48, pp. 13480-13489, 2015.

[49] S. J. Wang, J. J. Zhang, H. Y. Yang, F. Wang, and S. T. Li, "Acupoint specificity on acupuncture regulation of hypothalamic- pituitary-adrenal cortex axis function," $B M C$ Complementary and Alternative Medicine, vol. 15, p. 87, 2015.

[50] L. Liu, X. F. Wu, X. N. Zheng et al., "[Effect of point-moxibustion and electroacupuncture on the expression of endothelial nitric oxide synthase mRNA and angiotensin 2 mRNA in gastric antrum in diabetic gastroparesis rats]," Zhen Ci Yan Jiu, vol. 42, no. 3, pp. 240-245, 2017.

[51] Y. Peng, Y.-P. Lin, F.-E. He, and S.-X. Yi, "Effect of electroacupuncture on gastric motility, expressions of ghrelin and GHSR mRNA in gastric antrum tissue of diabetic gastroparesis rats," Journal of Acupuncture and Tuina Science, vol. 15, no. 2, pp. 88-93, 2017.

[52] J. W. Yang, Y. Peng, H. J. Chen et al., "[Effect of electroacupuncture intervention on gastrointestinal motility and expression of insulin-like growth factor 1 and its receptor proteins in gastric antrum in diabetic gastroparesis rats]," Zhen Ci Yan Jiu, vol. 42, no. 4, pp. 315-320, 2017.

[53] L. Chao, W. Yuan, X. Bin, and Y. Zhi, "Effect of acupuncture at three different acupoints on electrical activity of gastric distention-affected neurons in rat medial vestibular nucleus," Journal of Traditional Chinese Medicine, vol. 38, no. 1, pp. 125-131, 2018.

[54] H. Li, T. He, Q. Xu et al., "Acupuncture and regulation of gastrointestinal function," World Journal of Gastroenterology, vol. 21, no. 27, p. 8304, 2015.

[55] M. Lu, C. Chen, W. Li, Z. Yu, and B. Xu, "EA at PC6 promotes gastric motility: role of brainstem vagovagal neurocircuits," Evidence-Based Complementary and Alternative Medicine, vol. 2019, Article ID 7457485, 10 pages, 2019.

[56] C.-Y. Yong, S. Chen, H. Chen et al., "Effects of needling acupoints at different nerve segments on oxytocin neurons in rat's hypothalamic paraventricular nucleus and intragastric pressure," Journal of Acupuncture and Tuina Science, vol. 17, no. 5, pp. 297-304, 2019. 